\title{
Primary synovial sarcoma of the kidney: A case report
}

\author{
ZIJIAN WANG, ZHAOHUI ZHONG, LIANG ZHU, WEI XIONG, CIZHONG PAN, \\ XINJUN WANG, ZHICHAO HUANG and XIAOKUN ZHAO
}

Department of Urology, The Second Xiangya Hospital, Central South University, Changsha, Hunan 410011, P.R. China

Received September 19, 2014; Accepted April 28, 2015

DOI: $10.3892 / 01.2015 .3744$

\begin{abstract}
Synovial sarcoma (SS) is a soft-tissue tumor with a unique $\mathrm{t}(\mathrm{X} ; 18)(\mathrm{p} 11.2 ; \mathrm{q} 11.2)$ chromosomal translocation that can be identified by polymerase chain reaction in tissue homogenates. Generally, the tumor occurs in the proximity of the joints. The current study presents the case of a 54-year-old female with primary SS (PSS) of the right kidney; this is an extremely rare tumor accounting for $<2 \%$ of malignant renal carcinomas. The patient presented with an interrupted right flank pain of 20 years duration, with a precipitating factor of a hematuria of 8 days. Abdominal computerized tomography (CT) scan revealed a heterogeneous, unclear-marginated soft tissue arising in the upper pole of the right kidney with solid, necrotic components and heterogeneous enhancement. Renal cell carcinoma was diagnosed pre-operatively. A right nephrectomy was performed, and the patient is currently free of metastasis 12 months after the surgery. The current treatment for renal SS includes surgical resection and ifosfamide-based chemotherapy. To the best of our knowledge, $<50$ cases have been reported in the English literature. Urologists should be aware of the possibility of malignancy in cystic renal masses and consider a diagnosis of SS. Along with the case report, the current study presents a literature review on the diagnosis and treatment of PSS.
\end{abstract}

\section{Introduction}

Synovial sarcoma (SS) originates primarily in the soft tissues, generally in the para-articular regions of the extremities. These tumors can, however, involve other unusual locations (1), such as the pleura, lungs, mediastinum and kidneys. Primary renal $\mathrm{SS}$ is a rare carcinoma that was first described by Argani et al in 1999 (2). This tumor presents a diagnostic dilemma, as it is extremely difficult to distinguish from other renal carcinomas, including metastatic sarcoma and renal cell carcinoma

Correspondence to: Professor Xiaokun Zhao or Professor Zhaohui Zhong, Department of Urology, The Second Xiangya Hospital, Central South University, 139 Renmin Road, Changsha, Hunan 410011, P.R. China

E-mail: xiaokunzhao@126.com

E-mail: zhaohuizhong2013@163.com

Key words: renal cell carcinoma, synovial sarcoma, SYT-SSX with sarcomatoid differentiation, which may have similar histological characteristics (3). Iacovelli et al (4) examined 64 cases of SS and the median overall survival time was 48 months; in addition, at the time of diagnosis, $98 \%$ of patients had clinical symptoms, $67 \%$ had pain, and $38 \%$ had hematuria. The primary therapy for renal synovial sarcoma is radical surgical resection. However, no consensus has been reached on the adjuvant treatment since at present only case reports have been published. The current study reports a case of primary SS (PSS) of the kidney and presents a review of the relevant literature.

\section{Case report}

A 54-year-old female presented with interrupted right flank pain that had been apparent for 20 years, precipitating with hematuria for 8 days. Abdominal computed tomography revealed a heterogeneous, soft-tissue mass with unclear margins, arising in the upper pole of the right kidney. The mass exhibited solid, necrotic components and heterogeneous enhancement. No evidence of renal vein, inferior vena or right atrial thrombosis was found. No local invasion or lymphadenopathy was identified (Fig. 1). The clinical and radiological diagnosis was consistent with renal cell carcinoma.

A right nephrectomy was subsequently performed. The resected specimen weighed $210 \mathrm{~g}$ and measured $13 \times 5 \times 3.5 \mathrm{~cm}$. Macroscopic examination of the specimen revealed a cystic $8 \times 8 \times 7-\mathrm{cm}$ tumor with soft, solid growth, which was yellow-brown tan in color, in the upper pole the kidney. Hemorrhage and necrosis were also observed (Fig. 2).

Microscopically, the tumor was composed of spindle cells arranged in intersecting fascicles, alternating with hypocellular areas (Fig. 3). Immunohistochemical study showed positive staining for epithelial membrane antigen (EMA; Fig. 4A), cytokeratin (CK; Fig. 4B), B-cell lymphoma 2 (Bcl-2; Fig. 4C), vimentin (Vim) and cluster of differentiation (CD)99, and no staining for S100, CD34, CD117, synaptophysin, mesothelial cells and calretinin, which are both mesothelial tissue markers. Molecular analysis demonstrated a translocation between the SYT gene on chromosome 18 and the $\mathrm{X}$ chromosome, which confirmed the diagnosis of SS. Reverse transcription-polymerase chain reaction analysis also detected mRNA expression of the SYT-SXX1 gene. According to the findings, a diagnosis of monophasic spindle cell SS was formed. The patient is currently free of local recurrence or metastasis at 12 months post-surgery. 


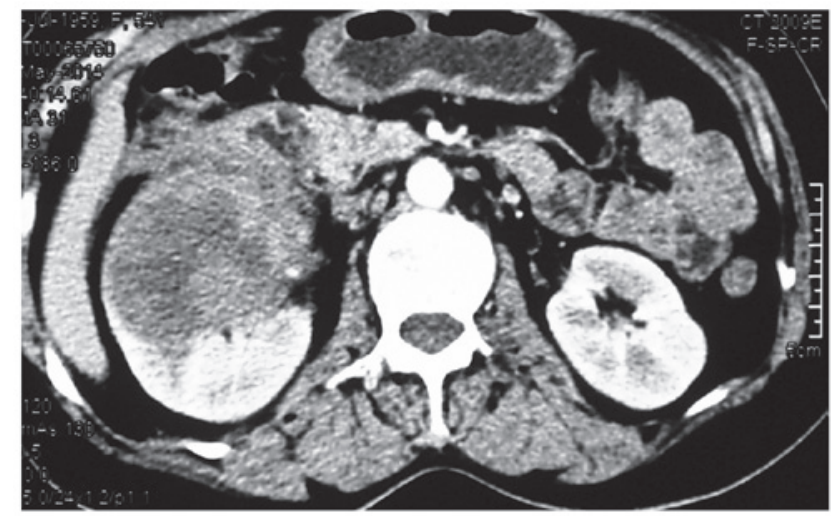

Figure 1. Abdominal computed tomography showing a soft-tissue mass in the right kidney.

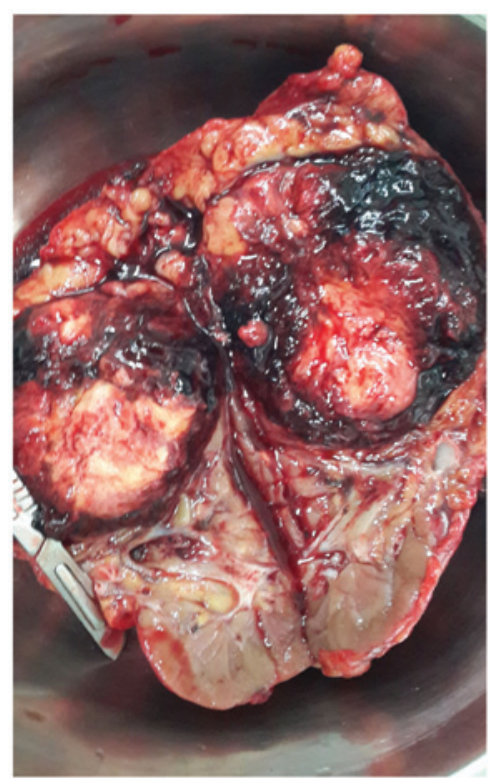

Figure 2. Restected 8x8x7-cm tumor following nephrectomy.

\section{Discussion}

PSS of the kidney is extremely rare and was first described by Argani et al in 2000 (2). To the best of our knowledge, $<50$ cases have been reported in the English literature. There is currently no standard treatment owing to the limited number of reported cases. Among sarcomas of the kidney, leiomyosarcoma is the most frequent type, comprising 40-60\%, followed by rhabdomyosarcoma, chondrosarcoma, liposarcoma, angiosarcoma, hemangiopericytoma and osteosarcoma (3).

Generally, SS affects adolescents and young adults between 20 to 50 years old (5). The mean age at diagnosis is 37 years (range, 13-67 years), $~ 1 \%$ of cases are located in the kidney. The mean tumor diameter is $11 \mathrm{~cm}$ (range, $3-21 \mathrm{~cm}$ ), and the rate of metastasis upon admission is likely to be low (5).

There are no clinical or imaging features that can indicate a diagnosis of renal SS. Thus, it is a challenge to diagnose the tumor due to its rarity and its similar presentation to other renal carcinomas $(6,7)$. Furthermore, renal SS is usually confused

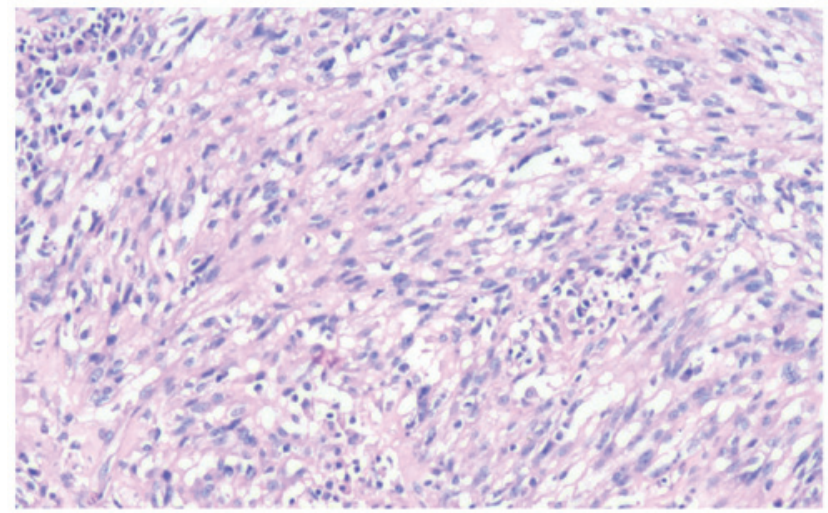

Figure 3. Tumor composed of spindle cells arranged in intersecting fascicles, alternating with hypocellular areas (hematoxylin and eosin staining; magnification, x100).

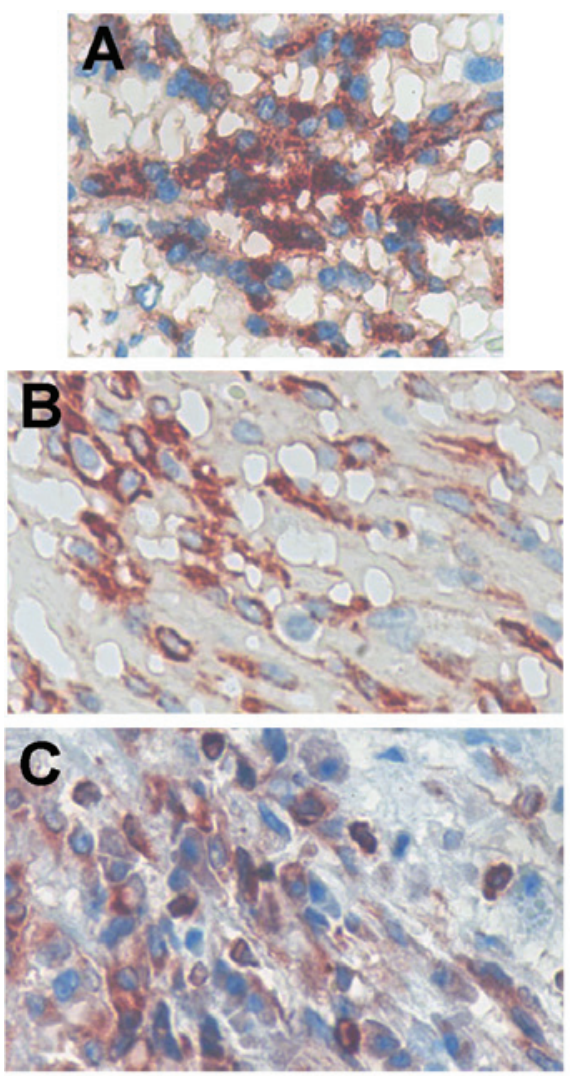

Figure 4. Immunohistochemical study of tumor cells showing positivity for (A) epthelial membrane antigen, (B) cytokeratin and (C) B-cell lymphoma 2 (stain, hematoxylin and eosin; magnification, x200).

with other soft-tissue sarcomas under the microscope. The majority of the tumors in the literature were initially interpreted as renal cell carcinoma. The differential diagnosis includes adult Wilms' tumors, transitional cell carcinoma, renal cell carcinoma, hemangiopericytoma and primitive neuroectodermal tumors (8).

Poorly-differentiated SS is formed from sheets of undifferentiated round cells with hyperchromatic nuclei and frequent mitoses, and is associated with the poorest outcome (9). A diagnosis of biphasic SS may be formed by the presence of both epithelial and spindle cell components, 
while monophasic SS is diagnosed with an epithelial or a spindle cell component only. However, the differentiation of monophasic SS from other spindle cell sarcomas may be difficult (5). Immunohistochemical studies can confirm the pathological diagnosis. SS usually stains positively for Bcl-2, CD99/Mic2, CD56, Vim and focally for EMA, but negatively for desmin, actin, WT-1, S-100, CD34 and CD31 $(5,9)$. The immunohistochemical results found in the present case were completely positive for Bcl-2, EMA and vimentin, focally positive for CD99, and completely negative for S-100, CD34 and CD117.

Currently, the gold standard for the diagnosis of synovial sarcoma is the fusion of the synovial sarcoma translocation, chromosome 18 gene (SYT; also known as SS18) on chromosome 18 to either the synovial sarcoma, X breakpoint 1 (SSX1) or SSX2 genes on chromosome Xp11 to form a SYT-SSX fusion $(8,10)$.

Although no guidelines have been established with regard to the treatment of primary renal SS due to the limited number of cases reported, surgery is considered the first choice. However, the prognosis is poor with the use of surgical treatment only. Based on observations that the tumor is more sensitive to chemotherapy than other soft-tissue sarcomas (11), chemotherapy is used in the clinic. The clinical benefit of adjuvant chemotherapy for sarcoma remains controversial, as there is no conclusive evidence that it confers any survival benefits. This may be due to the different histological subtypes that are reported in studies, as well as the evolution in the quality of treatment and the range of criteria that are used for the selection of patients for adjuvant systemic therapy (12). Due to the rarity of the tumor and the lack of any established benefits of adjuvant therapy in the literature, the present case used follow-up examinations only, with no adjuvant therapy. The patient is currently free of local recurrence or metastasis at 12 months post-surgery.

In conclusion, primary renal SS is an extremely rare neoplasm, with histomorphological and immunohistochemical features that may be confused with other spindle cell tumors of the kidney. The differential diagnosis of such a tumor is therefore difficult, and molecular or cytogenetic analysis should be used to confirm the pathological diagnosis. Although the clinical benefit of adjuvant chemotherapy remains uncertain, a multidisciplinary approach to treat the disease may bring some benefits to the patient, such as pain relief.

\section{References}

1. Divetia M, Karpate A, Basak R and Desai SB: Synovial sarcoma of the kidney. Ann Diagn Pathol 12: 333-339, 2008.

2. Argani P, Faria PA, Epstein JI, et al: Primary renal synovial sarcoma: Molecular and morphologic delineation of an entity previously included among embryonal sarcomas of the kidney. Am J Surg Pathol 24: 1087-1096, 2000.

3. Zakhary MM, Elsayes KM, Platt JF and Francis IR: Magnetic resonance imaging features of renal synovial sarcoma: A case report. Cancer Imaging 8: 45-47, 2008.

4. Iacovelli R, Altavilla A, Ciardi A, et al: Clinical and pathological features of primary renal synovial sarcoma: analysis of 64 cases from 11 years of medical literature. BJU Int 110: 1449-1154, 2012.

5. Ozkan EE, Mertsoylu H and Ozardali HI: A case of renal synovial sarcoma treated with adjuvant ifosfamide and doxorubicin. Intern Med 50: 1575-1580, 2011.

6. Dassi V, Das K, Singh BP and Swain SK: Primary synovial sarcoma of kidney: A rare tumor with an atypical presentation. Indian J Urol 25: 269-271, 2009.

7. Schaal CH, Navarro FC and Moraes Neto FA: Primary renal sarcoma with morphologic and immunohistochemical aspects compatible with synovial sarcoma. Int Braz J Urol 30: 210-213, 2004.

8. Koyama S, Morimitsu Y,Morokuma F and Hashimoto H: Primary synovial sarcoma of the kidney: Report of a case confirmed by molecular detection of the SYT-SSX2 fusion transcripts. Pathol Int 51: 385-391, 2001.

9. Nishida T, Inamoto $\mathrm{T}$, Uehara $\mathrm{H}$, et al: Monophasic primary renal synovial sarcoma accompanied with a hemorrhagic cyst. Urol J 8: 244-247, 2011

10. Gabilondo F, Rodríguez F, Mohar A, et al: Primary synovial sarcoma of the kidney: Corroboration with in situ polymerase chain reaction. Ann Diagn Pathol 12: 134-137, 2008.

11. Karavasilis V, Seddon BM, Ashley S, et al: Significant clinical benefit of first-line palliative chemotherapy in advanced soft-tissue sarcoma: retrospective analysis and identification of prognostic factors in 488 patients. Cancer 112: 1585-1591, 2008.

12. Blay JY and Le Cesne A: Adjuvant chemotherapy in localized soft tissue sarcomas: Still not proven. Oncologist 14: 1013-1020, 2009. 